\title{
Explaining, not just predicting, drives interest in personal genomics
}

\author{
Susanne F. Meisel', Deanna Alexis Carere ${ }^{2,3}$, Jane Wardle', Sarah S. Kalia ${ }^{4}$, Tanya A. Moreno ${ }^{5}$, Joanna L. Mountain ${ }^{6}$, \\ J. Scott Roberts ${ }^{7 \dagger}$, Robert C. Green ${ }^{2,8,9^{*}+}$ and for the PGen Study Group
}

\begin{abstract}
Background: There is a widespread assumption that risk prediction is the major driver of customer interest in personal genomic testing (PGT). However, some customers may also be motivated by finding out whether their existing diseases have a genetic etiology. We evaluated the impact of an existing medical diagnosis on customer interest in condition-specific results from PGT.
\end{abstract}

Methods: Using a prospective online survey of PGT customers, we measured customer interest prior to receiving PGT results for 11 health conditions, and examined the association between interest and personal medical history of these conditions using logistic regression.

Results: We analyzed data from 1,538 PGT customers, mean age 48.7 years, $61 \%$ women, $90 \%$ White, and $47 \%$ college educated. The proportion of customers who were 'very interested' in condition-specific PGT varied considerably, from $28 \%$ for ulcerative colitis to $68 \%$ for heart disease. After adjusting for demographic and personal characteristics including family history, having a diagnosis of the condition itself was significantly associated with interest in genetic testing for risk of that condition, with odds ratios ranging from $2.07(95 \% \mathrm{Cl} 1.28-3.37)$ for diabetes to 19.99 (95 \% Cl 4.57-87.35) for multiple sclerosis.

Conclusions: PGT customers are particularly interested in genetic markers for their existing medical conditions, suggesting that the value of genetic testing is not only predictive, but also explanatory.

\section{Background}

Direct-to-consumer (DTC) personal genomic testing (PGT) continues to be controversial, with debate centering on the analytical validity of PGT results, and the clinical validity and utility of returning this type of information to individual customers without involvement of healthcare providers [1-5]. Critics of PGT have cautioned that customers may misinterpret their genetic test results, leading to harm or unnecessary medical expenditure $[1,6]$. However, other commentators have emphasized the potential for PGT to raise awareness of genetic disease predisposition and prompt lifestyle changes to mitigate disease risk; ultimately reducing the burden posed by these conditions on the healthcare system $[7,8]$.

\footnotetext{
* Correspondence: rcgreen@genetics.med.harvard.edu

${ }^{\dagger}$ Equal contributors

2Division of Genetics, Department of Medicine, Brigham and Women's Hospital and Harvard Medical School, 75 Francis St, Boston, MA 02115, USA

${ }^{8}$ Harvard Medical School, Boston, MA 02115, USA

Full list of author information is available at the end of the article
}

Previous empirical research on the PGT consumer experience has focused primarily on the psychological and behavioral effects of PGT $[9,10]$. Somewhat less attention has been paid to the motivations of individuals who seek PGT. In the few studies that have investigated this topic, the predominant drivers of interest in PGT have been identified as curiosity and gaining insight into future disease risk [10-12]. However, alternative motivations may also exist: for example, in two qualitative studies, we found that overweight and obese individuals sought genetic testing in part to obtain an explanation for their condition $[13,14]$. As these findings were in a small sample, focused on only one health condition (obesity) and did not specifically pertain to DTC testing, it is not clear whether they could have application to the wider PGT consumer population.

In the traditional clinic setting, genetic testing related to a current diagnosis is frequently ordered for the purpose of identifying or confirming etiology, guiding treatment, determining prognosis, or providing recurrence 
risk information to families. To date, however, no quantitative study has investigated the effect of an existing medical diagnosis on interest in obtaining diseasespecific genetic risk information in the DTC PGT setting. Using data from the Impact of Personal Genomics (PGen) Study, [15] a longitudinal survey study of customers from 23andMe, Inc. [16] and Pathway Genomics, [17] we evaluated the association between personal history of a disease, family history of a disease, and interest in PGT results for that disease. Defining personal and demographic correlates of interest in obtaining PGT is important because these could influence subsequent affective and behavioral responses to test results $[10,12]$. Based on previous findings related to interest in genetic testing for obesity risk $[13,14]$, we hypothesized that PGT customers would be particularly interested in receiving genetic risk information for a health condition they already had, possibly even more than for a condition they might develop in the future.

\section{Materials and methods Study design}

Data for our analysis were obtained from the Impact of Personal Genomics (PGen) Study, a longitudinal study of DTC PGT customers from 23andMe and Pathway Genomics. The PGen Study protocol was designed and administered by academic researchers at Harvard Medical School/Brigham and Women's Hospital and the University of Michigan School of Public Health, in partnership with scientists from the two PGT companies, and independent survey design experts from SoundRocket (formerly Survey Sciences Group, Ann Arbor, MI, USA). Details of this academiaindustry collaboration, and the analytical design and administration of the PGen Study, have been reported previously $[15,18]$.

Briefly, new DTC PGT customers of 23andMe and Pathway Genomics were recruited via email between March and July 2012. Survey data were collected online at three time points: first after customers purchased PGT, but before they received their results (baseline; $\mathrm{BL})$, then approximately 2 weeks post results $(2 \mathrm{~W})$, and finally approximately 6 months post results (6M). A total of 1,648 participants completed the BL survey. PGT results were returned to customers as per standard company practice, and then linked to survey data at the end of survey administration. The PGen Study was approved by the Partners Human Research Committee and the University of Michigan Health Sciences and Behavioral Sciences Institutional Review Board, and informed consent was obtained electronically from each participant prior to enrollment. The research conformed to the Helsinki Declaration.

\section{Outcome variables}

For each condition addressed in the results report, customers of 23andMe and Pathway were provided with a personal risk estimate based on one or more single nucleotide polymorphism (SNP) markers. Interest in learning their risk estimate for each of 24 conditions was evaluated at BL with the multiple-choice question: 'How interested are you in learning about your genetic risk for each of these diseases', for which response options were: 'not at all interested/somewhat interested', and 'very interested'.

Whether or not a customer had ever had a diagnosis of each condition for which a risk estimate could be obtained from PGT was evaluated at BL with the question: 'Has a doctor ever told you that you have one of the following medical conditions'. Using a hierarchical question structure, participants were first presented with eight broad disease categories (neurological, psychiatric, gastrointestinal, heart, eye, diabetes, obesity, cancer), and then a choice of specific conditions for any selected category (for example, diabetes: Type 1 diabetes; Type 2 diabetes). They could select as many conditions as applicable.

Family history for each of the 24 conditions was evaluated at BL with the question: 'Have any of your blood relatives (a parent, brother or sister, child, grandparent, aunt, uncle, or first cousin) ever had any of the following conditions'. The same hierarchical question structure (broad then specific) was used to identify family history.

The following demographic characteristics were recorded at BL: age, gender, race/ethnicity (six race categories, plus Hispanic/Latino ethnicity option), and highest education level (11 categories). Current health status was assessed at BL with one multiple-choice question from the Control Preference Scale [19]: 'In general, would you say your health is: [excellent/very good/good/ fair/poor]'. State anxiety, which may affect health information seeking, [20] was evaluated at BL with the twoitem brief Generalized Anxiety Disorder screen (GAD-2) [21]. Multiple-choice responses to the two questions ('Over the past two weeks, how often have you felt nervous, anxious, or on edge' and 'Over the past two weeks, how often have you been unable to stop or control worrying') used response options from 'not at all' (scored 0) to 'nearly every day' (scored 3). A summed score of 4 or more represents a positive screen for generalized anxiety or panic disorder.

\section{Statistical analyses}

Our analysis used survey data from only the BL survey, which comprised 240 questions, including demographic characteristics, interest, and motivations for undergoing PGT, and personal and family history. For the purposes of our analysis, we restricted the PGen Study dataset to 
the 1,648 participants who completed the BL survey prior to viewing their results. We further excluded participants with missing data on the predictor variables. Descriptive statistics were computed to summarize the baseline demographic characteristics of the study sample, interest in PGT results for each condition, and the frequency of personal and family history of each condition. Descriptive statistics were also computed within each company-specific sample to investigate differences in characteristics between participants recruited from each PGT company.

Of the 24 conditions for which customers of both companies were provided with PGT results, 13 conditions (age-related macular degeneration, Alzheimer's disease, amyotrophic lateral sclerosis, blood clotting, breast cancer, celiac disease, chronic kidney disease, colorectal cancer, glaucoma, leukemia, lung cancer, Parkinson's disease, and prostate cancer) had a reported frequency of $<2 \%$. These conditions were excluded from analysis because of the small sample sizes. Results for the following 11 conditions are reported here: asthma, bipolar disorder, type 2 diabetes, heart disease (specifically coronary artery disease), high cholesterol, multiple sclerosis, obesity, osteoarthritis, rheumatoid arthritis, skin cancer, and ulcerative colitis.

We used multivariate logistic regression to evaluate associations between personal history of a condition, family history of a condition, and interest in conditionspecific PGT results. In all models, we dichotomized the outcome as 'very interested' vs. 'somewhat/not at all interested'. We used binary variables for 'ever' personal or family history of each condition, and models were adjusted for variables likely to be associated with interest based on prior work $[22,23]$, including age (continuous), gender (male vs. female), race (White vs. non-White), Hispanic/Latino ethnicity (yes vs. no), education (four categories, as described in Table 1), positive GAD-2 screen for anxiety/panic disorder (yes vs. no), ever history of any other condition (yes vs. no), and current health status (five categories, as reported). Finally, all analyses were adjusted for PGT company (23andMe vs. Pathway) to account for the different recruitment strategies employed by each company and the resulting differences in demographic and motivational characteristics within the company-specific sub-groups. Data were analyzed using the Statistical Package for Social Sciences, SPSS version 21.0 (Chicago, IL, USA).

\section{Results}

Of the 1,648 participants who completed the BL survey prior to viewing their results, 110 (6.7 \%) were excluded due to missing data necessary for our analyses. Demographic characteristics of the remaining 1,538 participants included in our analyses are shown in Table 1.
Table 1 Participant characteristics $(n=1,538)$

\begin{tabular}{ll}
\hline Variable & Frequency \\
& $\mathrm{n}(\%)$ \\
\hline Mean age (SD; range) & $48.7(15.5 ; 20-95)$ \\
Female & $936(60.9)$ \\
Non-White race & $154(10.0)$ \\
Hispanic/Latino ethnicity & $84(5.5)$ \\
23andMe customers & $978(63.6)$ \\
Positive GAD-2 screen for anxiety/panic disorder & $163(10.6)$ \\
Highest level of education & \\
$<$ College degree & $340(22.1)$ \\
College degree & $472(30.7)$ \\
Some graduate school & $531(34.5)$ \\
Doctoral degree & $295(12.7)$ \\
Self-reported health & \\
Poor & $65(4.2)$ \\
Fair & $172(11.2)$ \\
Good & $465(30.3)$ \\
Very good & $604(39.3)$ \\
Excellent & $231(15.0)$ \\
\hline
\end{tabular}

GAD-2, Generalized Anxiety Disorder - 2 Item Scale; SD, standard deviation

Compared to participants recruited from 23andMe, participants from Pathway were more likely to be women $(67.3 \%$ vs. $57.3 \%, P<0.001)$; to have a positive screen for anxiety at baseline $(13.8 \%$ vs. $8.8 \%, P=0.002)$; to report lower levels of education (global $F$-test $P<0.001$ ) and lower self-reported health (global $F$-test $P<0.001$ ); and tended to be younger ( mean age $=44.3$ vs. 51.2 , $P<0.001$ ) (Additional file 1: Table S1). The proportion of participants who reported that they were 'very interested' in PGT results for each of the 11 conditions ranged from $28.0 \%$ (ulcerative colitis) to $68.1 \%$ (heart disease) (Fig. 1). A majority of participants were very interested in results for high cholesterol, type 2 diabetes, skin cancer, and heart disease. Only 227 participants $(14.8 \%)$ were very interested in PGT for all 11 conditions, and even fewer $(\mathrm{n}=29,1.9 \%)$ stated that they were not at all interested in PGT for any of the 11 conditions. Figure 1 shows the proportion of participants who were very interested in PGT results for each condition, stratified by personal diagnosis status. Across all conditions, participants who reported a personal diagnosis of a given condition were more likely to be very interested in PGT results for that condition than participants who did not report it. Condition-specific interest was consistently higher among participants from Pathway, but no clear pattern of differences in frequency of personal diagnosis and family history was noted across companies (Additional file 1: Table S2). 


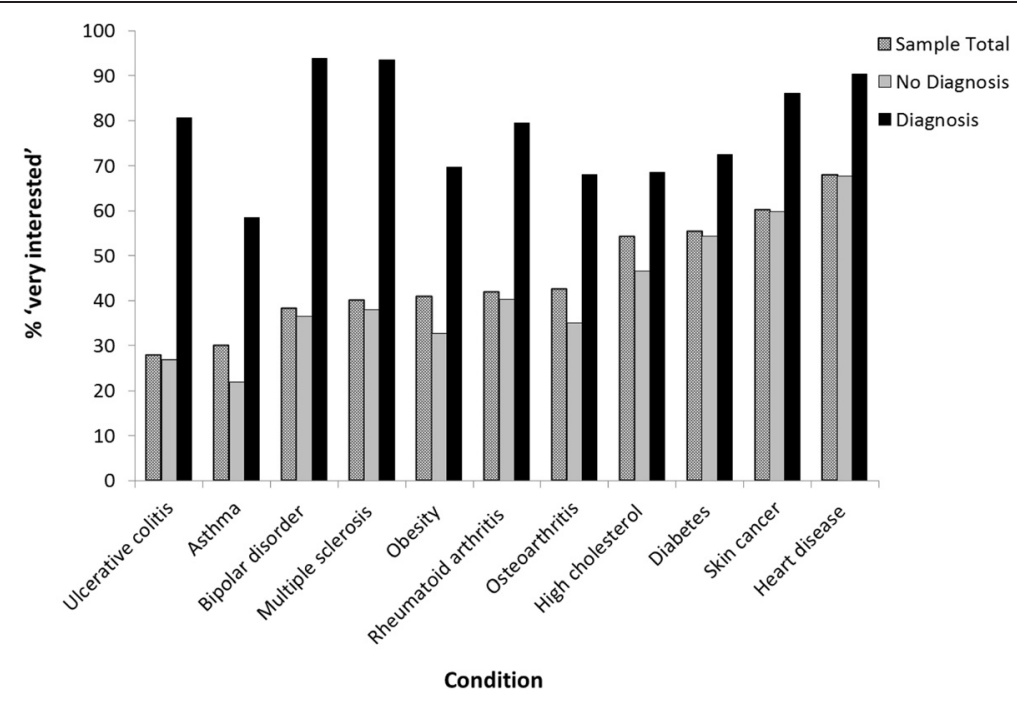

Fig. 1 Proportion of individuals 'very interested' in genetic testing for a condition they already have versus those without a diagnosis of the condition

The proportion of participants who reported a personal diagnosis of each of the 11 conditions ranged from $2.0 \%$ (heart disease, ulcerative colitis) to $36.2 \%$ (high cholesterol) (Table 2). Only five conditions (asthma, obesity, diabetes, osteoarthritis, high cholesterol) had a frequency greater than $15 \%$. The frequency of reported family history of each condition was typically higher, ranging from $6.9 \%$ (multiple sclerosis) to $68.6 \%$ (high cholesterol) (Table 2).

Results of the logistic regression analyses are presented in Table 2. After adjustment for age, gender, race, ethnicity, education, anxiety, PGT company, and diagnosis of any other condition, both personal and family history of a condition were significantly associated in all cases with interest in PGT results for that condition. Odds ratios for the effect of a personal history on interest ranged from 2.07 (diabetes) to 19.99 (multiple sclerosis), while odds ratios for the effect of a family history on interest were in most cases smaller, ranging from 1.55 (rheumatoid arthritis) to 4.14 (multiple sclerosis).

\section{Discussion}

Using baseline data from the PGen Study, we demonstrated that consumer interest in genetic risk information from PGT is independently associated both with having a family history of the condition and with having the condition itself. This finding is surprising because the emphasis of PGT marketing and discussion is typically focused on predicting future health risks. Although earlier work has often focused on the role of family history of a disease as motivation to obtain PGT $[11,12]$, the notion that DTC customers could be most interested in information on the etiology of their own disease condition has not yet been raised in discussions about the utility of PGT.
Comparisons of odds ratios across conditions encourage the speculation that rarer conditions, and conditions without firmly established etiology (such as multiple sclerosis, bipolar disorder, and ulcerative colitis), could evoke more interest in a genetic explanation than more common conditions (obesity, elevated cholesterol, and heart disease). Thus, it is possible that individuals who decide to purchase PGT anticipate deriving psychological benefit from information that might appear to shed light on the etiology of a disease they already have. Even for a condition that is as common as obesity, and where strong public beliefs about etiology exist, overweight participants in our prior qualitative studies described relief of guilt and self-blame after learning about their increased genetic risk of obesity, without any negative impact on motivation to battle their biology' [13, 14]. These findings match those from the area of genetic testing for mental health conditions, which also show that awareness of genetic risk may help to relieve rumination about causes of mental illness [24]. Alternatively, it is possible that individuals want to receive information on a genetic contribution to their personal illness in order to share this information with children or other family members for the purposes of risk evaluation and disease prevention [11, 12]. A third possibility is that customers believe knowledge about the underlying genetics of a condition could improve treatment outcomes, perhaps through pharmacogenomics. Since this is the first large-scale study to suggest an explanatory value of PGT for an existing condition, the findings need to be replicated and it will be important to carry out further qualitative and quantitative research to explore the reasons behind this phenomenon. 
Table 2 Individuals with personal diagnosis and family history of each condition, and logistic regression examining the association with interest among customers

\begin{tabular}{|c|c|c|c|c|}
\hline Condition & n (\%) & $O R^{a}$ & $95 \% \mathrm{Cl}$ & $P$ value \\
\hline \multicolumn{5}{|l|}{ Ulcerative colitis } \\
\hline No diagnosis/No FH & & 1 & & \\
\hline Diagnosis & $31(2.0)$ & 6.83 & $2.21-21.12$ & 0.001 \\
\hline $\mathrm{FHx}$ & $93(9.0)$ & 2.54 & $1.59-4.30$ & $<0.001$ \\
\hline \multicolumn{5}{|l|}{ Asthma } \\
\hline No diagnosis/No FH & & 1 & & \\
\hline Diagnosis & $341(22.2)$ & 2.86 & $1.66-4.90$ & $<0.001$ \\
\hline $\mathrm{FHx}$ & $552(36.5)$ & 1.82 & $1.43-2.31$ & $<0.001$ \\
\hline \multicolumn{5}{|l|}{ Bipolar disorder } \\
\hline No diagnosis/No FH & & 1 & & \\
\hline Diagnosis & $48(3.1)$ & 13.54 & $1.74-105.15$ & 0.013 \\
\hline $\mathrm{FHx}$ & $226(19.9)$ & 3.23 & $2.29-4.54$ & $<0.001$ \\
\hline \multicolumn{5}{|l|}{ Multiple sclerosis } \\
\hline No diagnosis/No FH & & 1 & & \\
\hline Diagnosis & $62(4.0)$ & 19.99 & $4.57-87.35$ & $<0.001$ \\
\hline $\mathrm{FHx}$ & $76(6.9)$ & 4.14 & $2.40-7.00$ & $<0.001$ \\
\hline \multicolumn{5}{|l|}{ Obesity } \\
\hline No diagnosis/No FH & & 1 & & \\
\hline Diagnosis & $350(22.8)$ & 2.35 & $1.17-5.4 .71$ & 0.016 \\
\hline $\mathrm{FHx}$ & $750(49.4)$ & 1.70 & $1.36-2.12$ & $<0.001$ \\
\hline \multicolumn{5}{|l|}{ Rheumatoid arthritis } \\
\hline No diagnosis/No FH & & 1 & & \\
\hline Diagnosis & $63(4.1)$ & 3.87 & $1.97-7.60$ & $<0.001$ \\
\hline $\mathrm{FHx}$ & $199(15.0)$ & 1.55 & $1.21-1.96$ & 0.005 \\
\hline \multicolumn{5}{|l|}{ Osteoarthritis } \\
\hline No diagnosis/No FH & & 1 & & \\
\hline Diagnosis & $353(23.0)$ & 2.51 & $1.63-3.87$ & $<0.001$ \\
\hline $\mathrm{FHx}$ & $593(44.7)$ & 1.49 & $1.17-1.90$ & 0.001 \\
\hline \multicolumn{5}{|l|}{ High cholesterol } \\
\hline No diagnosis/ No FH & & 1 & & \\
\hline Diagnosis & $556(36.2)$ & 2.98 & $1.86-4.76$ & $<0.001$ \\
\hline $\mathrm{FHx}$ & $1039(68.6)$ & 1.64 & $1.31-2.06$ & $<0.001$ \\
\hline \multicolumn{5}{|l|}{ Diabetes } \\
\hline No diagnosis/No FH & & 1 & & \\
\hline Diagnosis & $94(6.1)$ & 2.07 & $1.28-3.37$ & 0.003 \\
\hline $\mathrm{FHx}$ & $604(39.3)$ & 2.43 & $1.97-2.99$ & $<0.001$ \\
\hline \multicolumn{5}{|l|}{ Skin cancer } \\
\hline No diagnosis/ No FH & & 1 & & \\
\hline Diagnosis & $36(2.3)$ & 4.49 & $1.47-13.69$ & 0.008 \\
\hline $\mathrm{FHx}$ & $282(18.3)$ & 2.36 & $1.74-3.20$ & $<0.001$ \\
\hline
\end{tabular}

Table 2 Individuals with personal diagnosis and family history of each condition, and logistic regression examining the association with interest among customers (Continued)

Heart disease (coronary artery)

\begin{tabular}{lllll} 
No diagnosis/No FH & 1 & & \\
Diagnosis & $31(2.0)$ & 3.99 & $1.18-2.17$ & 0.018 \\
FHx & $575(56.7)$ & 2.10 & $1.66-2.62$ & $<0.001$ \\
\hline
\end{tabular}

${ }^{a}$ The model for each condition includes history of personal diagnosis and family history of that condition, and is adjusted for age, sex, race, ethnicity, education, company from which the test was purchased along with self-reported health and diagnosis of any other medical condition

Our findings are also consistent with several previous studies that have demonstrated that genetic testing for a particular condition is motivated by family history of that condition $[10,12]$. For example, a systematic review of 23 studies investigating uptake for breast cancer predictive genetic testing, either through actual uptake or anticipated uptake in hypothetical scenarios, found that individuals with a family history of cancer were twice as likely to have genetic testing for cancer risk as those without a family history [25]. Likewise, in a population-based survey, family history of heart disease was highly predictive of interest in genetic testing for heart disease [26].

This analysis had several strengths. The extensive information on demographics and family history collected in the PGen Study allowed us to control for possible confounding by the factors most commonly cited as having an impact on interest in genetic testing. The number of participants with each diagnosis was high enough to conduct the analysis, and we observed the effect regardless of condition, which increased our confidence in the validity of the findings. However, the study also had important limitations. Due to the small numbers of participants with a prior diagnosis for some of the diseases, confidence intervals were very wide for some conditions. Further research is needed to clarify whether the effect will also be observed in samples where a greater proportion has been diagnosed. Interest in PGT for specific conditions was only assessed with a simple three-point ordinal scale, without considering follow-up questions about its motivations or origins of interest. Future research could include questions that examine this topic in greater detail; for example, by using open-ended questions. For the current analyses, we only looked at 'ever' diagnosis of a condition, not whether customers were currently suffering with this condition. This may explain why a large proportion of participants reported both 'excellent' health, and having a medical diagnosis. Since a subset of PGen Study participants were drawn from a health-based social networking site, it is likely that these participants were particularly interested in exploring their health condition in more depth than the average 
person. Because participation was voluntary and part of a research study, our participants may differ from the typical PGT customer, and will certainly differ from the general population. Finally, all of our data were collected using self-report, which has been shown to vary in accuracy [27] and may introduce an element of bias.

\section{Conclusions}

In conclusion, data from the PGen Study showed that a prior diagnosis of a disease is significantly associated with interest in PGT for that condition, even after adjusting for variables commonly associated with increased interest in genetic testing; including family history. This correlation with interest in genetic testing has not been previously described in research on the benefits and harms of PGT, and it may be an important factor in evaluating the clinical and personal utility of personal genomic testing.

\section{Additional file}

Additional file 1: Tables S1 and S2 contain descriptive statistics stratified by personal genomic testing company. (DOCX $19 \mathrm{~kb}$ )

\section{Competing interests}

JLM is employed by 23andMe. TAM was employed by Pathway Genomics at the time of manuscript publication, and is now employed by Millennium Health. The remaining authors declare that they have no competing interests. Although industry partners were collaborators in the current study, they did not provide any funding or other financial contribution.

\section{Authors' contributions}

SFM conceived of the analysis, analyzed the data, interpreted the data, and drafted the manuscript; DAC participated in study design, helped with data interpretation, and critically revised the manuscript; JW conceived of the analysis, helped with data interpretation, and critically revised the manuscript: SSK, TAM, and JLM participated in the design of the PGen Study, helped with data acquisition, and critically revised the manuscript; JSR and RCG conceived of the PGen Study, participated in questionnaire design, conceived of the analysis, helped with data interpretation, and critically revised the manuscript. All authors have seen and approved the final manuscript.

\section{Acknowledgements \\ The PGen Study is supported by the National Institutes of Health $(\mathrm{NIH})$ National Human Genome Research Institute (R01-HG005092). SFM is supported by a grant from Cancer Research UK (C1418/A10843). DAC is supported by the Canadian Institutes of Health Research (Doctoral Foreign Study Award). RCG is also supported by NIH U01-HG006500, U19-HD077671, and R01-HG002213. The content is solely the responsibility of the authors and does not necessarily represent the official views of the National Center for Research Resources, the National Institutes of Health, or the Canadian Institutes of Health Research.}

\section{Members of the PGen study team}

Members of the PGen Study at the time of publication are as follows: Robert C Green, Joel B Krier, Caroline M Weipert, Sarah S Kalia, Kurt D Christensen, Lisa S Lehmann, Harvard Medical School and Brigham and Women's Hospital; Deanna Alexis Carere, Peter Kraft, Harvard T.H. Chan School of Public Health; J Scott Roberts, Mack T Ruffin IV, Lan Q Le, Jenny Ostergren, University of Michigan School of Public Health; Wendy R Uhlmann, Mick P Couper, University of Michigan; Joanna L Mountain, Amy K Kiefer, 23andMe; Tanya A Moreno, Adrian Vilalta, Pathway Genomics; Scott D Crawford, SoundRocket, formerly Survey Sciences Group; L Adrienne Cupples, Clara A Chen, Catharine Wang, Boston University; Stacy W Gray, Dana-Farber Cancer Institute; Barbara A Koenig, University of California San Francisco; Kimberly Kaphingst, University of Utah; Sarah Gollust, University of Minnesota.

\section{Author details}

${ }^{1}$ Cancer Research UK Health Behaviour Research Centre, Department of Epidemiology and Public Health, University College London, London, UK. 2Division of Genetics, Department of Medicine, Brigham and Women's Hospital and Harvard Medical School, 75 Francis St, Boston, MA 02115, USA. ${ }^{3}$ Program in Genetic Epidemiology and Statistical Genetics, Department of Epidemiology, Harvard T.H. Chan School of Public Health, Boston, MA 02115 USA. ${ }^{4}$ Icahn Institute for Genomics and Multiscale Biology, Icahn School of Medicine at Mount Sinai, New York, NY 10029, USA. ${ }^{5}$ Millennium Health, San

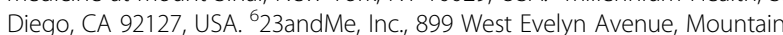
View, CA 94041, USA. 'Department of Health Behavior \& Health Education, University of Michigan School of Public Health, Ann Arbor, MI 48109, USA. ${ }^{8}$ Harvard Medical School, Boston, MA 02115, USA. ${ }^{9}$ Partners Personalized Medicine, EC Alumnae Building, Suite 301, 41 Avenue Louis Pasteur, Boston, MA 02115, USA

Received: 20 March 2015 Accepted: 15 June 2015

Published online: 01 August 2015

\section{References}

1. Frueh FW, Greely HT, Green RC, Hogarth S, Siegel S. The future of direct-toconsumer clinical genetic tests. Nat Rev Genet. 2011;12:511-5.

2. Evans JP, Green RC. Direct to consumer genetic testing: avoiding a culture war. Genet Med. 2009;11:568-9.

3. Caulfield T, McGuire AL. Direct-to-consumer genetic testing: perceptions, problems, and policy responses. Annu Rev Med. 2012;63:23-33.

4. Evans JP, Meslin EM, Marteau TM, Genomics CT. Deflating the genomic bubble. Science. 2011;331:861-2.

5. Roberts JS, Ostergren J. Direct-to-consumer genetic testing and personal genomics services: a review of recent empirical studies. Curr Genet Med Rep. 2013;1:182-200.

6. Khoury MJ, McBride CM, Schully SD, loannidis JP, Feero WG, Janssens AC, et al. The scientific foundation for personal genomics: recommendations from a National Institutes of Health-Centers for Disease Control and Prevention multidisciplinary workshop. Genet Med. 2009;11:1-9.

7. Collins FS. The Human Genome Project and the future of medicine. Ann N Y Acad Sci. 1999:882:42-55. discussion 56-65.

8. Green RC, Farahany NA. Regulation: the FDA is overcautious on consumer genomics. Nature. 2014;505:286-7.

9. Bloss CS, Schork NJ, Topol EJ. Effect of direct-to-consumer genomewide profiling to assess disease risk. N Engl J Med. 2011;364:524-34.

10. Kaufman DJ, Bollinger JM, Dvoskin RL, Scott JA. Risky business: risk perception and the use of medical services among customers of DTC personal genetic testing. J Genet Couns. 2012;21:413-22.

11. McGowan ML, Fishman JR, Lambrix MA. Personal genomics and individual identities: motivations and moral imperatives of early users. New Genet Soc. 2010;29:261-90.

12. Su Y, Howard HC, Borry P. Users' motivations to purchase direct-toconsumer genome-wide testing: an exploratory study of personal stories. J Community Genet. 2011;2:135-46.

13. Meisel SF, Wardle J. Responses to FTO genetic test feedback for obesity in a sample of overweight adults: a qualitative analysis. Genes Nutr. 2014;9:374.

14. Meisel SF, Wardle J. 'Battling my biology': psychological effects of genetic testing for risk of weight gain. J Genet Couns. 2014;23:179-86.

15. Carere DA, Couper MP, Crawford SD, Kalia SS, Duggan JR, Moreno TA, et al. Design, methods, and participant characteristics of the Impact of Personal Genomics (PGen) Study, a prospective cohort study of direct-to-consumer personal genomic testing customers. Genome Med. 2014;6:96.

16. 23andMe. Available at: https://www.23andme.com/.

17. Pathway Genomics. Available at: http://www.pathway.com.

18. Lehmann LS, Kaufman DJ, Sharp RR, Moreno TA, Mountain JL, Roberts JS, et al. Navigating a research partnership between academia and industry to assess the impact of personalized genetic testing. Genet Med. 2012;14:268-73.

19. Degner LF, Sloan JA, Venkatesh P. The Control Preferences Scale. Can J Nurs Res. 1997;29:21-43

20. Case DO, Andrews JE, Johnson JD, Allard SL. Avoiding versus seeking: the relationship of information seeking to avoidance, blunting, coping, dissonance, and related concepts. J Med Libr Assoc. 2005:93:353-62.

21. Donker T, van Straten A, Marks I, Cuijpers P. Quick and easy self-rating of Generalized Anxiety Disorder: validity of the Dutch web-based GAD-7 GAD-2 and GAD-SI. Psychiatry Res. 2011;188:58-64. 
22. Sanderson SC, O'Neill SC, Bastian LA, Bepler G, McBride CM. What can interest tell us about uptake of genetic testing? Intention and behavior amongst smokers related to patients with lung cancer. Public Health Genomics. 2010;13:116-24.

23. Graves KD, Peshkin BN, Luta G, Tuong W, Schwartz MD. Interest in genetic testing for modest changes in breast cancer risk: implications for SNP testing. Public Health Genomics. 2011;14:178-89.

24. Potokar DN, Stein CH, Darrah OA, Taylor BC, Sponheim SR. Knowledge and attitudes about personalized mental health genomics: narratives from individuals coping with serious mental illness. Community Ment Health J. 2012;48:584-91.

25. Ropka ME, Wenzel J, Phillips EK, Siadaty M, Philbrick JT. Uptake rates for breast cancer genetic testing: a systematic review. Cancer Epidemiol Biomarkers Prev. 2006;15:840-55.

26. Sanderson SC, Wardle J, Jarvis MJ, Humphries SE. Public interest in genetic testing for susceptibility to heart disease and cancer: a population-based survey in the UK. Prev Med. 2004;39:458-64.

27. Stone AA, Bachrach CA, Jobe JB, Kurtzman HS, Cain VS. The science of selfreport: implications for research and practice. 1st ed. Hove: Psychology Press; 1999.

\section{Submit your next manuscript to BioMed Central and take full advantage of:}

- Convenient online submission

- Thorough peer review

- No space constraints or color figure charges

- Immediate publication on acceptance

- Inclusion in PubMed, CAS, Scopus and Google Scholar

- Research which is freely available for redistribution 